\title{
Kisspeptin signalling linked to obesity
}

New research in mice shows that targeted inactivation of the kisspeptin receptor gene (Kiss1r) results in obesity and a diabetic phenotype. The findings demonstrate novel metabolic roles of kisspeptin signalling that might be relevant to obesity, diabetes and broader metabolic dysfunction in humans.

The neuropeptide kisspeptin stimulates gonadotropin-releasing hormone neurons in the brain-a key step in activation of the reproductive axis. Evidence of widespread expression of kisspeptin and its receptor in metabolic tissues, such as fat, liver and pancreas, led the researchers to study the energetic and metabolic phenotype of mice deficient in kisspeptin signalling.

Kiss $1 r^{-/}$female mice exhibited increased body weight, adiposity and leptin levels, and reduced glucose tolerance, compared with wild-type sex-matched littermates; conversely, male $\mathrm{Kiss} \mathrm{r}^{-/-}$mice had normal body weight and glucose tolerance. Female transgenic mice consumed less food than their wild-type counterparts, however, they were still obese-possibly owing to observed reductions in respiratory rate, energy expenditure and locomotor activity. The results demonstrate that kisspeptin signalling influences energy and metabolic status in a gender-specific manner.

"These findings might have important implications for understanding the mechanisms that underlie obesity and diabetes in humans," explains lead investigator Alexander Kauffman. "Future studies will be aimed at determining where in the body the metabolic effects of kisspeptin signalling occur and if similar phenotypes are present in humans lacking functional kisspeptin and/or its receptor."

\section{David Holmes}

Original article Tolson, K. P. et al. Impaired kisspeptin signaling decreases metabolism and promotes glucose intolerance and obesity. J. Clin. Invest. doi:10.1172/ JCI71075 